\title{
Wavevector filtering through single-layer and bilayer graphene with magnetic barrier structures
}

\author{
M. Ramezani Masir, ${ }^{1, a)}$ P. Vasilopoulos, ${ }^{2}$ and F. M. Peeters ${ }^{1}$ \\ ${ }^{1}$ Department of Physics, University of Antwerp, Groenenborgerlaan 171, B-2020 Antwerpen, Belgium \\ ${ }^{2}$ Department of Physics, Concordia University, Montreal, Quebec H3G 1M8, Canada
}

(Received 15 July 2008; accepted 25 November 2008; published online 15 December 2008)

\begin{abstract}
We show that the angular range of the transmission through magnetic barrier structures can be efficiently controlled in single-layer and bilayer graphenes and this renders the structure's efficient wavevector filters. As the number of magnetic barriers increases, this range shrinks, the gaps in the transmission versus energy become wider, and the conductance oscillates with the Fermi energy. (C) 2008 American Institute of Physics. [DOI: 10.1063/1.3049600]
\end{abstract}

Graphene's electronic properties are drastically different from those, say, of conventional semiconductors. Charge carriers in a wide single-layer graphene behave like "relativistic," chiral massless particles with a "light speed" equal to the Fermi velocity and possess a gapless, linear spectrum close to the $K$ and $K^{\prime}$ points. ${ }^{1}$ A perfect transmission through arbitrarily high and wide barriers, referred to as Klein tunneling, ${ }^{2}$ is expected. In contrast, carriers in bilayer graphene possess a quadratic spectrum with zero gap. However, applying a gate allows the opening of a gap due to the tunnel coupling between the layers. This impedes the Klein tunneling and is more appropriate for certain applications, e.g., for improving the on/off ratio in carbon-based transistors. A recent review of the properties of graphene is given in Ref. 3.

An angular confinement of the transmission in singlelayer graphene was reported in Refs. 4 and 5 using magnetic barrier structures. However, in none of these works was the wavevector filtering behavior of magnetic barriers addressed. In this letter we show how this angular confinement can be maximized, in single-layer and bilayer graphenes, and detail the resulting filtering behavior. This occurs for finite-width and $\delta$-function barriers but not for complex magnetic structures in which the average magnetic field is zero.

An electron in single-layer graphene in the presence of a perpendicular magnetic field $B(x)$, which varies along the $x$ direction, is described by the $2 \times 2$ Hamiltonian $H_{0}$ $=v_{F} \boldsymbol{\sigma} \cdot[\mathbf{p}+e \mathbf{A}(x)]$. A solution of Dirac's equation $H_{0} \Psi$ $=E \Psi$, in regions where the magnetic field is not zero, is a linear combination of Weber functions. ${ }^{4,5}$ For an electron in bilayer graphene, the relevant Hamiltonian is a $4 \times 4$ matrix ${ }^{5,6}$ with elements $H_{13}=H_{31}=t$, where $t$ is the tunnel coupling strength that is assumed constant $t \approx 400 \mathrm{meV}$. Using the dimensionless units $B(x) \rightarrow B_{0} B(x), A(x) \rightarrow B_{0} \ell_{B} A(x)$, $\vec{r} \rightarrow \ell_{B} \vec{r}, \vec{v} \rightarrow v_{F} \vec{v}, E \rightarrow E_{0} E$, and $t \rightarrow E_{0} t$, with $E_{0}=\hbar v_{F} / \ell_{B}$ and $\ell_{B}=\left[\hbar / e B_{0}\right]^{1 / 2}$, the energy spectrum for a homogeneous magnetic field takes the form

$$
E_{n, \pm}= \pm\left[2 n+1+t^{2} / 2 \pm \sqrt{t^{4} / 4+(2 n+1) t^{2}+1}\right]^{1 / 2} .
$$

For $t=0$ it gives the spectrum for single-layer graphene ${ }^{6}$ $E= \pm[2(n+1)]^{1 / 2}$. The low-energy spectra for a barrier on single-layer and bilayer graphenes are partly shown in the

${ }^{a)}$ Electronic mail: mrmphys@gmail.com. lower panel of Fig. 1 as a function of $k_{y}$. For single-layer graphene in a region of homogeneous magnetic field, the wave function, up to a normalization factor, is written in terms of the Weber functions $D_{p}(z), p=E^{2} / 2$, as

$$
\Phi(z) \sim\left[\begin{array}{c}
-i(E / \sqrt{2}) D_{p-1}(z) \\
D_{p}(z) .
\end{array}\right] .
$$

For bilayer graphene the corresponding wave function is

$$
\Phi^{ \pm}(z) \sim\left[\begin{array}{c}
D\left(p^{ \pm}, z\right) \\
\left(-i \frac{\sqrt{2} p^{ \pm}}{E}\right) D\left(p^{ \pm}-1, z\right) \\
\left(\frac{E}{t}-\frac{2 p^{ \pm}}{t E}\right) D\left(p^{ \pm}, z\right) \\
i \frac{\sqrt{2}}{E}\left(\frac{E}{t}-\frac{2 p^{ \pm}}{t E}\right) D\left(p^{ \pm}+1, z\right)
\end{array}\right],
$$

where $\quad p^{ \pm}=\left(\gamma_{ \pm}-1\right) / 2, \quad \gamma_{ \pm}=E^{2} \pm\left(1+E^{2} t^{2}\right)^{1 / 2}, \quad$ and $z=2^{1 / 2}\left(x+k_{y}\right)$. In regions where the magnetic field is zero, the solution is written as ( $T$ denotes the transpose)
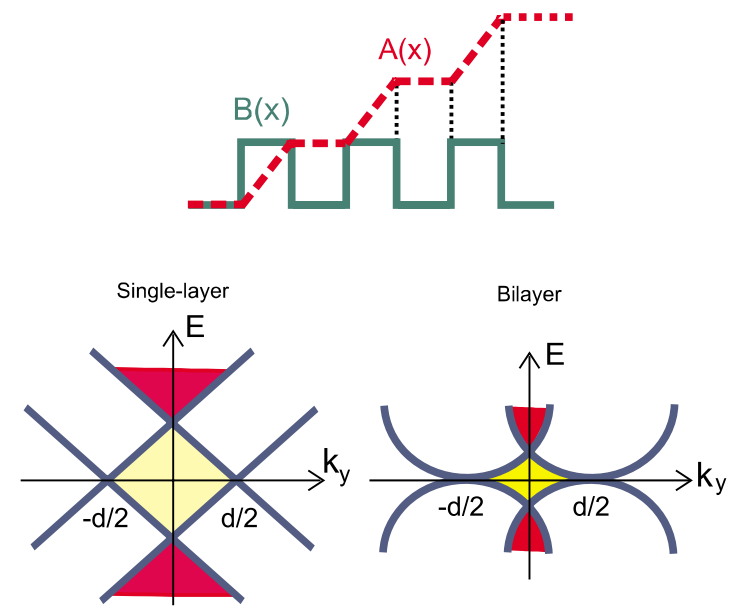

FIG. 1. (Color online) Top panel: magnetic field profile $B(x)$ and corresponding vector potential $A(x)$ for a series of barriers. Lower panel: a map in $\left(E, k_{y}\right)$ space, showing the regions in which tunneling involving real wavevectors is allowed (in red) and in which is not (in yellow) for one magnetic barrier in single-layer and bilayer graphene. Bound states can be found in the yellow regions delimited by the free-particle spectra (Ref. 5). 


$$
\Psi_{E, \pm}^{R}=N_{ \pm}\left(\mp E, \mp k_{x}^{ \pm} \pm i k_{y}, E, k_{x}^{ \pm}+i k_{y}\right)^{T} e^{i k_{x}^{ \pm} x+i k_{y} y},
$$

for waves propagating to the right, and as

$$
\Psi_{E, \pm}^{L}=N_{ \pm}\left(\mp E, \pm k_{x}^{ \pm} \pm i k_{y}, E,-k_{x}^{ \pm}+i k_{y}\right)^{T} e^{-i k_{x}^{ \pm} x+i k_{y} y},
$$

for waves propagating to the left. In this case the energy spectrum and wave vector $k_{x}^{ \pm}$are

$$
\begin{aligned}
& E=\mp t / 2 \pm\left[t^{2} / 4+K^{ \pm}\right]^{1 / 2}, \\
& k_{x}^{ \pm}=\left[E^{2}-k_{y}^{2} \pm E t\right]^{1 / 2},
\end{aligned}
$$

where $K^{ \pm}=k_{x}^{2 \pm}+k_{y}^{2}$. The factor $N_{ \pm}=1 /\left(4 W E k_{x}^{ \pm}\right)^{1 / 2}$ can be obtained from the normalization of the current. To evaluate the transmission through one or many barriers, we use the transfer-matrix technique and at each interface we match the wave function and the flux. ${ }^{5}$ Scattering between the $K$ and $K^{\prime}$ valleys is negligible for fields below $10^{4} \mathrm{~T} .^{7}$ For a single barrier we can evaluate the angular confinement by imposing the condition that the wave number $k_{x}$ be real for incident and transmitted waves. This gives

$$
\begin{aligned}
& k_{i}=\left[E^{2}-\left(k_{y}+A_{i}\right)^{2}\right]^{1 / 2}, \\
& k_{i}^{ \pm}=\left[(E \pm t / 2)^{2}-t^{2} / 4-\left(k_{y}+A_{i}\right)^{2}\right]^{1 / 2},
\end{aligned}
$$

respectively, for a single layer and a bilayer. Further, $i=1,2$, $A_{1}=d / 2$, and $A_{2}=-d / 2$. From Eqs. (8) and (9) and the relations $k_{y}=E_{F} \sin \theta$ for a single layer and $k_{y}$ $=\left(E_{F}^{2} \pm E_{F} t\right)^{1 / 2} \sin \theta^{ \pm}$for a bilayer, we can find the range of the angle of incidence $\theta$ in which the transmission is confined. For a single barrier we obtain

$$
-1 \leq \sin \theta \leq 1-d / E,
$$

in single-layer graphene, and

$$
-1 \leq \sin \theta^{ \pm} \leq 1-d /\left(E^{2} \pm E t\right)^{1 / 2},
$$

in bilayer graphene. In single-layer graphene the angle of exit $\theta^{\prime}$ is related to $\theta$ by

$$
\sin \theta^{\prime}=\sin \theta+d / E
$$

and in bilayer graphene by

$$
\sin \theta^{\prime \pm}=\sin \theta^{ \pm}+d /\left(E^{2} \pm E t\right)^{1 / 2} .
$$

We can also replace $\sin \theta$ by $k_{y} / k$ in Eqs. (10)-(13). If we do not use the dimensionless units, the last terms in Eqs. (10)-(13) are multiplied by $\lambda=\hbar v_{F} / \ell_{B}^{2}$, i.e.,

$$
d / E \rightarrow \lambda d / E, \quad d /\left(E^{2} \pm E t\right)^{1 / 2} \rightarrow \lambda d /\left(E^{2} \pm E t\right)^{1 / 2} .
$$

From Eqs. (10) and (12) one can find the limits for $\theta^{\prime}$. Notice that Eq. (10) implies a total reflection for $d>2 E$. We remark in passing that for the usual two-dimensional Schrödinger electrons, Eqs. (10) and (12) remain valid with $E$ replaced by $(2 E)^{1 / 2}$.

By controlling the ratio $d / E$ or $d /\left(E^{2} \pm E t\right)^{1 / 2}$, Eqs. (10) and (11) show how one can control the angular range of the transmission. For instance, one could double the width of the barrier or raise $E_{F}$, through the density, and/or $t$ by doping ${ }^{8}$ or gating. ${ }^{9}$ However, one may not have a good continuous control over these parameters, e.g., over the width $d$, which may be limited by fabrication techniques. It is therefore worth searching for other ways to control these parameters.

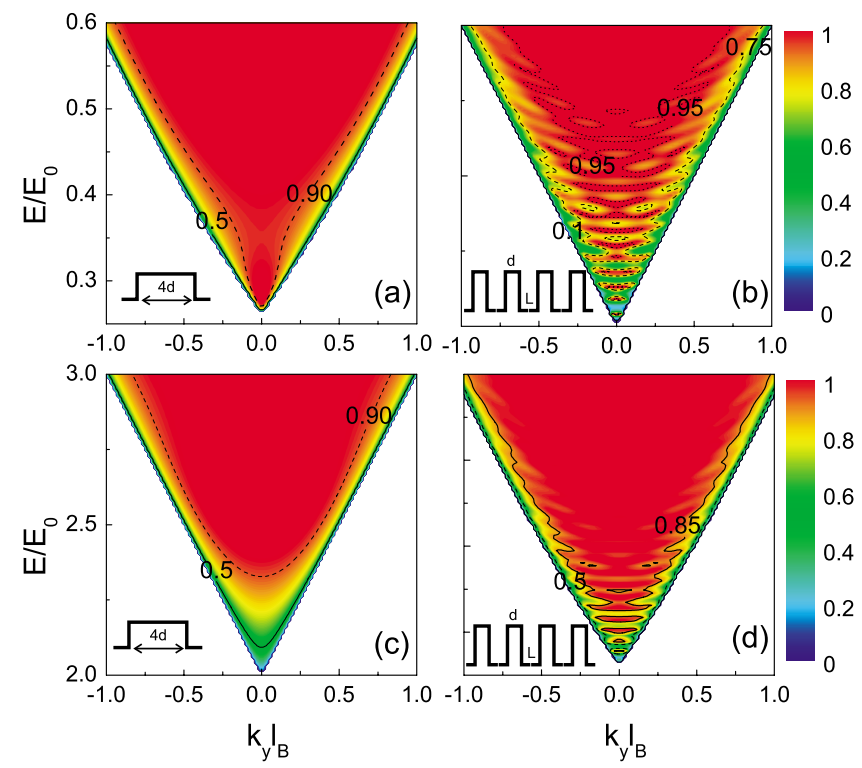

FIG. 2. (Color online) Contour plot of the transmission through one magnetic barrier of width $d=4 \ell_{B}$ in (a) and through four barriers of width $d=\ell_{B}$ in (b) for single-layer graphene. (c) and (d), respectively, as in (a) and (d) for bilayer graphene with $t=15$.

With this in mind we investigate the effect of increasing the number of barriers on the transmission. The region in which tunneling, involving real wavevectors $k_{x}$, is allowed is shaded in red in Fig. 1. For $n$ barriers the angular range is given by Eqs. (10) and (11) upon changing $d$ to $n d$. From Eqs. (10) and (11) we can evaluate the minimum energy required for tunneling. For $n$ barriers of width $d$ and $\theta=0$, we have $E=n d / 2$ for single-layer graphene and $E$ $=\mp(t / 2) \pm(t / 2)\left[1+n^{2} d^{2} / t^{2}\right]^{1 / 2}$ for bilayer graphene. For $t \gg n d$ we can rewrite this minimum as $E \approx \pm n^{2} d^{2} / 4 t$. This shows clearly that we can control the tunneling by increasing the number of barriers. Typically the height of the magnetic barriers is in the range of $0.1-1 \mathrm{~T}$. For $B=1 \mathrm{~T}$ the magnetic length is $l_{b}=25.6 \mathrm{~nm}$ and the energy scale is $E_{0}$ $=25.7 \mathrm{meV}$ for a typical $v_{F}=10^{6} \mathrm{~m} / \mathrm{s}$. In Fig. 2 we show a contour plot of the transmission, in $\left(E, k_{y}\right)$ space, for one barrier of width $d=4 \ell_{B}$ and for four magnetic barriers of width $d=\ell_{B}$. As seen using four barriers instead of one results in sharp resonances shown in the lower part of the right panels for both single-layer and bilayer graphenes. This holds for any multibarrier structure $n>2$.

As Eq. (10) shows, the angular range of the transmission shrinks by increasing the number of barriers. We show this in Fig. 3(a) for bilayer graphene and in Fig. 3(b) for singlelayer graphene. Had we used one barrier of width $d=4 \ell_{b}$, the shrinking of the shown angular range would be the same but the resonances at the lower left end of the plot would be absent. This can be seen more clearly in Fig. 4 where we plot the transmission versus the energy for $\theta=-\pi / 4$ and different numbers of barriers. Notice that the energy gap widens with $n$. Having seen the transmission resonances, one may wonder as to what extent they survive the averaging over the Fermi surface that determines the conductance $G$, which is more easily accessed in experiments than the transmission. For very low temperatures, such that $f(E)-f(E+e V) \approx-e V \delta(E$ $-E_{F}$ ), with $f(E)$ as the Fermi-Dirac function and $e V$ as the source-to-drain voltage drop, the standard formula for $G$ is $G=G_{0} \int_{-\pi / 2}^{\pi / 2} T\left(E_{F}, E_{F} \sin \theta\right) \cos \theta d \theta$, with $G_{0}=2 e^{2} E_{F} L_{y} / \pi h$ 

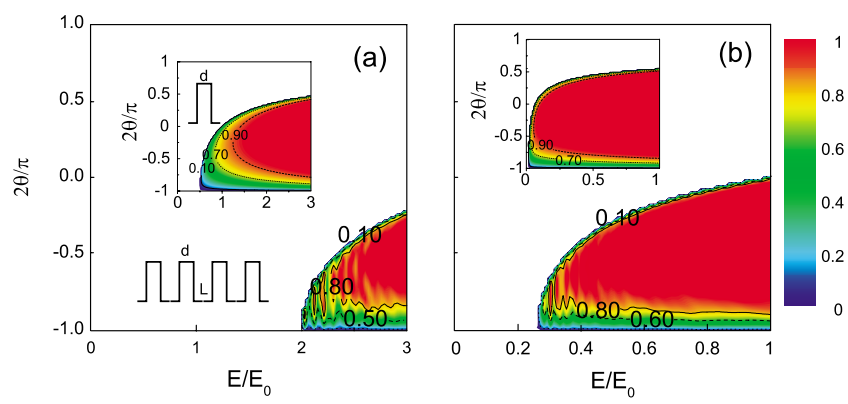

FIG. 3. (Color online) (a) Contour plot of the transmission in $(E, \theta)$ space for four barriers of width $d=\ell_{B}$ with $L=10 \ell_{B}$ in bilayer graphene. The inset shows the result for one barrier with $d=\ell_{B}$. (b) As in (a) but for single-layer graphene.

and $L_{y}$ as the length of the structure along the $y$ direction. We plot the conductance of a four-barrier structure in singlelayer graphene in Fig. 5(a), and in bilayer graphene in Fig. 5(b) with $d=\ell_{B}$ and two values of the interbarrier distance, $L=10 \ell_{B}$ and $L=20 \ell_{B}$, and $t=15$. The insets show the corresponding results for a single barrier of width $d=4 \ell_{B}$. As shown, in line with the transmission results of Fig. 3, those for the conductance show oscillations in the multibarrier structure that are absent in the single barrier.

In summary, we showed that the angular range of the transmission through magnetic barrier structures can be effi-

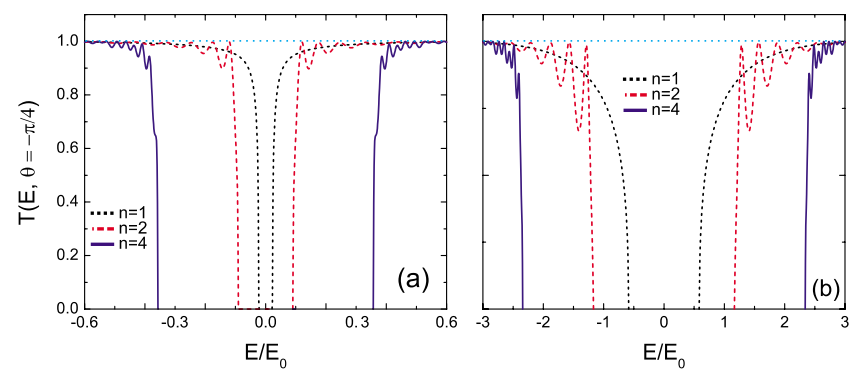

FIG. 4. (Color online) Transmission probability for different numbers of magnetic barriers in bilayer graphene (a) and single-layer graphene (b) with the parameters in Fig. 3.
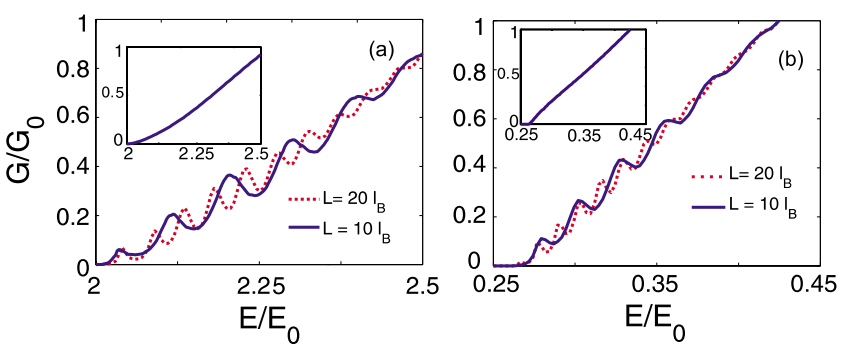

FIG. 5. (Color online) Conductance through four magnetic barriers of width $d=\ell_{B}$ in single-layer graphene (a) and in bilayer graphene (b) for two values of $L$ and $t=15$. The upper insets show the result through one magnetic barrier of width $d=4 \ell_{B}$.

ciently controlled by increasing the number of barriers in single-layer and bilayer graphenes. This renders the structure's efficient wavevector filters, leads to transmission resonances and conductance oscillations, and widens the gaps in the transmission as a function of the energy.

This work was supported by the Flemish Science Foundation (FWO-Vl), the Belgian Science Policy (IAP), and the NSERC Grant No. OGP0121756.

${ }^{1}$ K. S. Novoselov, A. K. Geim, S. V. Morozov, D. Jiang, Y. Zhang, S. V. Dubonos, I. V. Grigorieva, and A. A. Firsov, Science 306, 666 (2004); Y. Zhang, Y. W. Tan, H. L. Stormer, and P. Kim, Nature (London) 438, 201 (2005).

${ }^{2}$ M. I. Katsnelson, K. S. Novoselov, and A. K. Geim, Nat. Phys. 2, 620 (2006); J. M. Pereira, Jr., V. Mlinar, F. M. Peeters, and P. Vasilopoulos, Phys. Rev. B 74, 045424 (2006).

${ }^{3}$ A. H. Castro Neto, F. Guinea, N. M. R. Peres, K. S. Novoselov, and A. K. Geim, Rev. Mod. Phys. 80 (2008).

${ }^{4}$ A. De Martino L. Dell' Anna, and R. Egger, Phys. Rev. Lett. 98, 066802 (2007).

${ }^{5}$ M. Ramezani Masir, P. Vasilopoulos, A. Matulis, and F. M. Peeters, Phys. Rev. B 77, 235443 (2008).

${ }^{6}$ J. Milton Pereira, Jr., F. M. Peeters, and P. Vasilopoulos, Phys. Rev. B 76, 115419 (2007).

${ }^{7}$ S. Park and H.-S. Sim, Phys. Rev. B 77, 075433 (2007).

${ }^{8}$ T. Ohta, A. Bostwick, T. Seyller, K. Horn, and E. Rotenberg, Science $\mathbf{3 1 3}$ 951 (2006).

${ }^{9}$ E. V. Castro, K. S. Novoselov, S. V. Morozov, N. M. R. Peres, J. M. B. Lopes dos Santos, J. Nilsson, F. Guinea, A. K. Geim, and A. H. Castro Neto, Phys. Rev. Lett. 99, 216802 (2007). 
Applied Physics Letters is copyrighted by the American Institute of Physics (AIP). Redistribution of journal material is subject to the AIP online journal license and/or AIP copyright. For more information, see http://ojps.aip.org/aplo/aplcr.jsp 